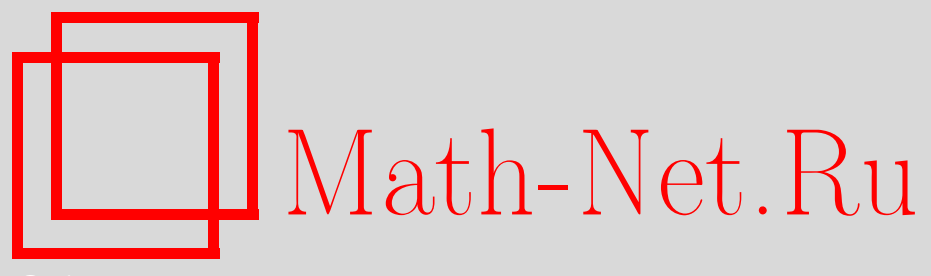

О. И. Мохов, Классификация многомерных скобок Пуассона гидродинамического типа, УМH, 2006, том 61, выпуск 2, 167-168

DOI: https://doi.org/10.4213/rm1724

Использование Общероссийского математического портала Math-Net.Ru подразумевает, что вы прочитали и согласны с пользовательским соглашением http://www . mathnet.ru/rus/agreement

Параметры загрузки:

IP: 18.234 .197 .8

26 апреля 2023 г., 16:01:42

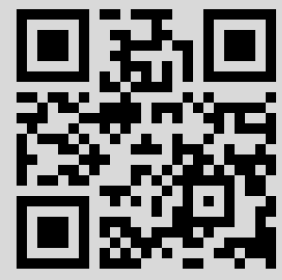




\section{Классификация многомерных скобок Пуассона гидродинамического типа}

\section{О. И. Мохов}

В данной работе решена хорошо известная проблема Дубровина-Новикова, поставленная еще в 1984 г., - проблема классификации многомерных скобок Пуассона гидродинамического типа, т.е. скобок Пуассона вида

$$
\left\{u^{i}(x), u^{j}(y)\right\}=\sum_{\alpha=1}^{n}\left(g^{i j \alpha}(u(x)) \delta_{\alpha}(x-y)+b_{k}^{i j \alpha}(u(x)) u_{\alpha}^{k}(x) \delta(x-y)\right),
$$

где $u=\left(u^{1}, \ldots, u^{N}\right)$ - локальные координаты на некотором гладком $N$-мерном многообразии $M$ или в области $\mathbb{R}^{N}, x=\left(x^{1}, \ldots, x^{n}\right), y=\left(y^{1}, \ldots, y^{n}\right)$ - независимые переменные, $g^{i j \alpha}(u), b_{k}^{i j \alpha}(u)$ - гладкие функции локальных координат $\left(u^{1}, \ldots, u^{N}\right)$, $1 \leqslant i, j, k \leqslant N, 1 \leqslant \alpha \leqslant n, u(x)=\left(u^{1}(x), \ldots, u^{N}(x)\right)$ - гладкие функции (поля) $n$ независимых переменных $x^{1}, \ldots, x^{n}$ со значениями в многообразии $M, u_{\alpha}^{k}(x)=\partial u^{k} / \partial x^{\alpha}$. Скобки Пуассона вида (1) были введены Б. А. Дубровиным и С. П. Новиковым в [1] (одномерный случай $n=1$ ) и [2] (многомерный случай) в связи с гамильтоновой теорией систем гидродинамического типа. В [1] получена полная классификация невырожденных одномерных скобок Пуассона вида (1), а в [2] поставлена задача классификации многомерных скобок Пуассона вида (1) и предпринята попытка получить классификацию этих скобок в первом нетривиальном случае $n=N=2$ (в [3] было показано, что этот результат работы [2] ошибочен). Классификация двухкомпонентных ( $N=2, n$ - любое) невырожденных скобок Пуассона вида (1) получена автором в [3], где получена также классификация для $1 \leqslant N \leqslant 4$, для чего пришлось развить мощный алгебраический аппарат. Решить задачу для произвольного числа компонент $N$ алгебраическими методами пока не удается. В данной работе дифференциально-геометрическими методами получена классификация всех неособых невырожденных многомерных скобок Пуассона вида (1) для любого $n$ и для любого числа компонент $N$. Напомним, что класс скобок вида (1) инвариантен относительно локальных замен координат $\left(u^{1}, \ldots, u^{N}\right)$. При этом коэффициенты скобки $g^{i j \alpha}(u)$ и $b_{k}^{i j \alpha}(u)$ преобразуются как дифференциально-геометрические объекты. Для любой скобки (1) коэффициенты $g^{i j \alpha}(u), 1 \leqslant \alpha \leqslant n$, являются контравариантными тензорами на многообразии $M$. Если $\operatorname{det}\left(g^{i j \alpha}(u)\right) \neq 0$ для всех $\alpha$, то скобка (1) называется невырожденной. В невырожденном случае можно ввести коэффициенты $\Gamma_{s k}^{j \alpha}(u)$ по формулам $b_{k}^{i j \alpha}(u)=-g^{i s \alpha}(u) \Gamma_{s k}^{j \alpha}(u)$. При этом коэффициенты $\Gamma_{s k}^{j \alpha}(u)$ преобразуются как коэффициенты аффинной связности на многообразии $M$. Для любой многомерной скобки Пуассона вида (1) для каждого $\alpha$ соответствующее слагаемое в правой части формулы (1) является одномерной скобкой Пуассона гидродинамического типа и в силу теоремы Дубровина-Новикова для невырожденных одномерных скобок Пуассона вида (1) [1] все невырожденные тензоры $g^{i j \alpha}(u)$ симметричны и являются плоскими метриками (метриками нулевой римановой кривизны), а каждая соответствующая аффинная связность $\Gamma_{s k}^{j \alpha}(u)$ согласована со своей метрикой $g^{i j \alpha}(u)$ и имеет нулевое кручение и нулевую риманову кривизну, т.е. аффинная связность $\Gamma_{s k}^{j \alpha}(u)$ является связностью Леви-Чивиты и однозначно определяется плоской метрикой $g^{i j \alpha}(u)$. Таким образом, любая невырожденная скобка Пуассона вида (1) однозначно определяется плоскими метриками $g^{i j \alpha}(u)$, которые в многомерном случае связаны дополнительными жесткими ограничениями.

Работа выполнена при финансовой поддержке Фонда содействия отечественной науке, Российского фонда фундаментальных исследований (гранты № 03-01-00782 и 05-01-00170) и программы поддержки ведущих научных школ (грант № 2185.2003.1). 
Теорема 1 [3]. Плоские невырожденные метрики $g^{i j \alpha}(u)$ определяют многомерную скобку Пуассона вида (1) тогда и толъко тогда, когда выполнены соотношения 1) $T^{i j k \alpha \beta}(u)=T^{k j i \alpha \beta}(u)$, 2) $\sum_{(i, j, k)} T^{i j k \alpha \beta}(u)=0$, 3) $T^{i j s \alpha \beta}(u) T_{s t}^{r \alpha \beta}(u)=$ $\left.T^{i r s \alpha \beta}(u) T_{s t}^{j \alpha \beta}(u), 4\right) \nabla_{r}^{\alpha} T^{i j k \alpha \beta}(u)=0$, где $T_{j k}^{i \alpha \beta}(u)=\Gamma_{j k}^{i \beta}(u)-\Gamma_{j k}^{i \alpha}(u)-$ npensmствующиц тензор, $T^{i j k \alpha \beta}(u)=-g^{k s \beta}(u) g^{i r \alpha}(u) T_{r s}^{j \alpha \beta}(u)$, знак $\sum_{(i, j, k)}$ означает суммирование по всем ииклическим перестановкам индексов $(i, j, k), \nabla_{r}^{\alpha}-\kappa о-$ вариантная производная, задаваемая связностъю $\Gamma_{j k}^{i \alpha}(u), \Gamma_{j k}^{i \alpha}(u)$ - связность Леви-Чивиты, порождаемая метрикой $g^{i j \alpha}(u)$.

Тензоры $T_{j k}^{i \alpha \beta}(u)$ являются препятствиями к приведению невырожденных многомерных скобок Пуассона вида (1) к постоянному виду, т.е. невырожденная скобка Пуассона вида (1) приводится локальной заменой координат к постоянной скобке тогда и только тогда, когда все препятствующие тензоры $T^{i j k \alpha \beta}(u)$ тождественно равны нулю. Напомним, что две псевдоримановы контравариантные метрики $g_{1}^{i j}(u)$ и $g_{2}^{i j}(u)$ называются согласованными, если для любой линейной комбинации этих метрик $g^{i j}(u)=\lambda_{1} g_{1}^{i j}(u)+\lambda_{2} g_{2}^{i j}(u)$, где $\lambda_{1}$ и $\lambda_{2}-$ произвольные константы, коэффициенты соответствующих связностей Леви-Чивиты и компоненты соответствующих тензоров римановой кривизны связаны тем же самым линейным соотношением: $\Gamma_{k}^{i j}(u)=\lambda_{1} \Gamma_{1, k}^{i j}(u)+\lambda_{2} \Gamma_{2, k}^{i j}(u), R_{k l}^{i j}(u)=\lambda_{1} R_{1, k l}^{i j}(u)+\lambda_{2} R_{2, k l}^{i j}(u)$ [4]. Теория согласованных метрик построена в работе автора [4].

Теорема 2 [5]. Метрики $g^{i j \alpha}(u)$, определяющие многомерную скобку Пуассона вида (1), согласованы.

Пара метрик $g_{1}^{i j}(u)$ и $g_{2}^{i j}(u)$ называется неособой, если собственные значения этой пары метрик, т.е. корни уравнения $\operatorname{det}\left(g_{1}^{i j}(u)-\lambda g_{2}^{i j}(u)\right)=0$, различны (ситуация пары метрик общего положения).

Теорема 3 [4]. Если пара метрик $g_{1}^{i j}(u)$ u $g_{2}^{i j}(u)$ является неособой, то эти метрики согласованы тогда и только тогда, когда тензор Нейенхейса аффинора $v_{j}^{i}(u)=g_{1}^{i s}(u) g_{2, s j}(u)$ тождественно равен нулю $\left(g_{2, s j}(u)\right.$ - ковариантная метри$\left.\kappa a: g_{2}^{i s}(u) g_{2, s j}(u)=\delta_{j}^{i}\right)$.

В этом случае существуют локальные координаты, в которых аффинор $v_{j}^{i}(u)$ является диагональным. В этих локальных координатах обе метрики $g_{1}^{i j}(u)$ и $g_{2}^{i j}(u)$ также обязаны быть диагональными. Более того, имеет место следующая важная теорема.

Теорема 4 [4]. Если пара метрик $g_{1}^{i j}(u)$ и $g_{2}^{i j}(u)$ является неособой, то эти метрики согласованы тогда и только тогда, когда существуют локальные координать $u=\left(u^{1}, \ldots, u^{N}\right)$ такие, что $g_{2}^{i j}(u)=g^{i}(u) \delta^{i j} u g_{1}^{i j}(u)=f^{i}\left(u^{i}\right) g^{i}(u) \delta^{i j}$, где $f^{i}\left(u^{i}\right)$, $i=1, \ldots, N,-$ функиии одной переменной.

Теорема 5. Если для невырожденной многомерной скобки Пуассона вида (1) одна из метрик $g^{i j \alpha}(u)$ образует неособые пары со всеми остальными метриками скобки, то эта скобка Пуассона приводится $к$ постоянному виду локальной заменой координат.

\section{Список литературы}

[1] Б. А. Дубровин, С.П. Новиков, Докл. АН СССР, 270:4 (1983), 781-785. [2] Б. А. Дубровин, С. П. Новиков, Докл. АН СССР, 279:2 (1984), 294-297. [3] О. И. Мохов, Функи. анализ и его прил., 22:4 (1988), 92-93. [4] О. И. Мохов, Функи. анализ и его прил., 35:2 (2001), 24-36. [5] О. И. Мохов, Симплектическая и пуассонова геометрия на пространствах петель гладких многообразий и интегрируемые уравнения, ИКИ, М.-Ижевск, 2004.

О. И. Мохов (О. I. Mokhov)

Центр нелинейных исследований при Институте теоретической физики им. Л. Д. Ландау РАН

E-mail: mokhov@mi.ras.ru, mokhov@bk.ru
Представлено С. П. Новиковым Принято редколлегией 21.12.2005 